\title{
La lingua della vita e la lingua della memoria
}

\author{
Julio Monteiro Martins
}

\section{OpenEdition}

\section{Journals}

Edizione digitale

URL: https://journals.openedition.org/cher/10963

DOI: 10.4000/cher.10963

ISSN: 2803-5992

\section{Editore}

Presses universitaires de Strasbourg

\section{Edizione cartacea}

Data di pubblicazione: 30 juin 2013

Paginazione: 63-68

ISBN: 978-2-35410-054-4

ISSN: 1968-035X

\section{Notizia bibliografica digitale}

Julio Monteiro Martins, "La lingua della vita e la lingua della memoria», reCHERches [Online], 10 | 2013, online dal 08 février 2022, consultato il 10 février 2022. URL: http://journals.openedition.org/cher/ 10963 ; DOI: https://doi.org/10.4000/cher.10963

\section{cc) (1) (ㅇ)}

Ce(tte) œuvre est mise à disposition selon les termes de la Licence Creative Commons Attribution Pas d'Utilisation Commerciale - Partage dans les Mêmes Conditions 4.0 International. 


\title{
La lingua della vita e la lingua della memoria
}

\author{
Julio Montelro Martins \\ Scrittore
}

\begin{abstract}
$\mathrm{N}$ ella mia lunga esperienza ho visto raramente un seminario con tanti scrittori riuniti allo stesso tempo e nello stesso luogo'. In Brasile un tale avvenimento è stato possibile solo durante il periodo della dittatura militare, perché poi gli scrittori erano diventati tutti un po' come delle star, dei divi, così succedeva che uno, invitato a un convegno, dicesse: «se c'è tizio, non vado» oppure «c'è troppa gente». Vorrei iniziare citando tre autori che in questi ultimi vent'anni di storia letteraria della migrazione italiana sono già scomparsi. Uno è Heleno Oliveira, brasiliano del Pernambuco, che viveva a Firenze e di cui Mia Lecomte ha curato una monografia molto bella. Oliveira era uno scrittore cristiano, cattolico, dalla sensibilità molto raffinata, universale. Ha viaggiato per varie zone dell'Africa facendo un lavoro interiore molto interessante sul modo in cui sentiva Firenze e l'Egitto. $\mathrm{Ci}$ sono delle poesie molto belle che mettono in rapporto questi due luoghi dell'anima. In seguito è stato a Lisbona nel 1995, ha incontrato una grande scrittrice portoghese, Sophia de Mello Breyner, sua amica, e mentre era lì ha avuto un infarto ed è morto all'età di quarant'anni. È morto proprio la stessa settimana in cui io arrivavo in Italia, e per di più avevamo la stessa età. Allora non riesco a liberarmi dal sospetto che sia morto proprio nel momento in cui ero presente io per raccogliere il testimone della staffetta. È una cosa di grande contenuto simbolico. L'altro è Thea Laitef, giovane scrittore iracheno,
\end{abstract}

1 Questo intervento è stato elaborato sotto forma di dialogo con Emanuele CutinelliRendina. 
poeta, presente peraltro nell'antologia $A i$ confini del verso ${ }^{2}$, un autore che è morto giovanissimo, che ha scritto delle poesie molto forti, potenti, anche perché era sconvolto dalle circostanze del suo esilio. E infine Egidio Molinas Leiva, scrittore paraguaiano, che ha scritto in italiano un romanzo molto bello, intitolato La notte del Yacarés. Lavorava a Roma come muratore. Egidio non era giovane, aveva già una sessantina di anni, ma aveva i propri genitori ancora vivi nel Paraguay e la cosa che più lo rattristava era il fatto di non poterli rivedere. Per questioni politiche non poteva entrare nel paese. L'ultima cosa che ha fatto in vita sua è stata quella di andare in Argentina, vicino al confine, per vedere se riusciva a rivedere $\mathrm{i}$ genitori prima che morissero. Loro sarebbero dovuti arrivare fino al confine del Paraguay con l'Argentina e attraversarlo. Ma non hanno avuto il permesso e l'incontro non è riuscito. Egidio è tornato a Roma molto deluso, depresso, e dopo poco è morto di un attacco di cuore. È stato seppellito in un tumulo anonimo. Una tomba senza lapide. Quindi non vorrei che questi tre poeti, scrittori e romanzieri migranti fossero assenti da queste pagine.

La lingua della vita e la lingua della memoria: in verità sono le lingue della memoria e nel mio caso sono almeno tre.

Una di queste è stata quella in cui durante un periodo di grande repressione e spavento ci arrivavano i libri degli scrittori sudamericani, non in lingua portoghese - gli scrittori di lingua portoghese non potevano scrivere, non potevano pubblicare - ma in lingua spagnola. Arrivavano dal Cile, molti scrittori vivevano lì, nel periodo del Presidente Salvador Allende, e poi da Cuba, Messico, Perù, Venezuela. E che cos'erano questi libri? Erano soprattutto raccolte di racconti brevi. Posso citare due autori che sono stati molto importanti nella mia formazione: Jorge Luis Borges e Julio Cortázar. Lo Spagnolo, più che la lingua della memoria, è stata per la mia generazione la lingua della libertà. Inoltre all'epoca c'erano ottime riviste letterarie, come ad esempio "Crisis», pubblicata a Buenos Aires dallo scrittore uruguaiano Eduardo Galeano, conosciuto in Italia per il saggio di grande successo Le vene aperte dell'America Latina ${ }^{4}$. Era il periodo dell'apice del racconto breve, di autori ispanoamericani di grande richiamo, come Garcia Márquez, ma

2 M. Lecomte, Ai confini del verso, Firenze, Le Lettere, 2006.

3 E. Molinas Leiva, La notte del Yacaré, Rep. di San Marino, Melting Pot, Guaraldi AIEP, 1998.

4 E. Galeano, Le vene aperte dell'America Latina, pref. di Isabel Allende, trad. di G. Lapasini e E. Liverano, Milano, Sperling \& Kupfer, 1997 (tit. orig. Las venas abiertas de América Latina). 
anche di molti altri meno conosciuti, e forse mai tradotti in Europa, come il narratore surrealista messicano Edmundo Valadés, direttore dell'importante rivista «El Cuento» e autore della straordinaria raccolta La muerte tiene permiso $^{5}$. La possibilità del racconto breve, nella situazione politica in cui vivevamo, riduceva i nostri rischi personali, perché aveva la virtù di poter essere letto più velocemente, e si poteva anche fare una semplice fotocopia e leggerlo in pubblico in due minuti e poi far scomparire i fogli. Facevamo a volte delle sessioni di letture letterarie clandestine, nello scantinato della facoltà di Comunicazione, a Niterói, o durante il Festival d'Inverno nella città barocca di Ouro Preto. Forse sarebbe più preciso dire che allora ho riscoperto il racconto breve, che conoscevo già, soprattutto dalla letteratura nord-americana - di cui mia madre era professoressa all'università - con autori come O'Henry, Poe, Hawthorne, Fitzgerald o Raymond Carver e John Cheever che all'epoca non erano ancora conosciuti fuori dagli Stati Uniti.

L'Inglese è stata la mia seconda lingua della memoria, soprattutto il «dolce Inglese» che mi arrivava attraverso la voce di mia madre - il che rende un po' più articolato il concetto di «lingua madre» -

E prima di parlare della terza lingua della memoria, vorrei aprire una parentesi per riflettere sul racconto breve. Il racconto, secondo me, è il genere della contemporaneità, perché è in grado di rispecchiare meglio di qualunque altro la sensibilità dell'uomo contemporaneo. Infatti, il romanzo classico, di stampo manzoniano, romanzo che ha dominato soprattutto alla fine dell'Ottocento e anche una gran parte del Novecento, rifletteva un certo tipo di visione del mondo nella quale la realtà si presentava in modo coerente, più intero, organico, spiegabile con un nesso causa-effetto $\mathrm{e}$ quindi al riparo dal caos, con gli eventi orchestrati in modo sequenziale o a volte circolare, ma sempre collegati tra di loro da una logica inflessibile. Un mondo facilmente comprensibile dai lettori, forse anche auspicabile, pieno di empatia, con affabulazioni che scorrevano con la regolarità di un grande fiume, ma un mondo ormai sempre più distante e distaccato dalle esperienze reali, vissute nel quotidiano dai lettori, prive di quella coerenza e carenti di senso, restie alla comprensione, persino impenetrabili e avverse a qualsiasi identificazione. In questo modo la lettura dei vecchi romanzi poteva portare un effetto consolatorio, anche se ingannevole, e convincere il lettore per un attimo che il mondo aveva la dimensione della sua ragione, che era un

5 E. Valadés, La muerte tiene permiso, Ciudad de Mexico, Fondo de Cultura Economica, 1955. 
paesaggio riconoscibile e che poteva essere catturato in una frazione di sguardo.

E invece il racconto breve ha abbandonato queste pretese onnicomprensive per assumere la frammentazione del reale e la precarietà dello sguardo soggettivo. E la frammentazione al contempo si è spostata dal racconto verso il romanzo contemporaneo, in una vera esplosione della sua architettura e della sua ideologia. E poi è venuto il cinema, e la televisione, e Internet, che non ci hanno portato soltanto una comunicazione intensa e un avvicinamento delle diverse parti del mondo, ci hanno portato anche una nuova forma direi di conoscenza epistemologica del mondo, a mosaico, a pezzettini. Ormai abbiamo una soggettività costruita attraverso lo zapping. Che cos'è lo zapping? È un cambiamento frenetico di canali televisivi, che produce un caleidoscopio di immagini e di emozioni disparate, e che succede anche quando sei collegato a Internet e clicchi su un sito e poi su un altro, e lasci aperte tutte le finestre, con voci, testi, suoni, immagini e melodie in contemporanea. La grande sfida dell'uomo contemporaneo, che non può più sfuggire a questo zapping, è riuscire all'interno dello zapping a identificare le differenze fondamentali e i valori, altrimenti si rischia che il mondo diventi un grande TG1, che la forma cancelli il contenuto, attraverso l'appiattimento assoluto di tutti gli argomenti: dalla tragedia del terremoto si passa ai cuccioli di orso che sono nati nello zoo di Londra o ai vantaggi del colesterolo «buono». Credo che un romanzo come il mio, madrelingua ${ }^{6}$, sia un esempio di narrativa che non sente nostalgia del romanzo tradizionale, che non rimpiange affatto l'agonia della vecchia struttura romanzesca, in favore della frammentazione, di una soggettività scoppiata, di una percezione discontinua del mondo e proprio per questo più aderente alla nostra realtà. È la storia di un romanzo che non vuole nascere, e che così rimane incompiuto, con diversi narratori che si contendono la narrazione, costruito a mosaico, con l'uso del tempo narrativo troppo esteso o troppo concentrato, che sembra persino non esistere, o essere bloccato, con al suo interno una strana enciclopedia arbitraria, e che alla fine, in una postfazione alla stessa opera, riesce a concludersi e chiude tutte le scatole rimaste aperte. In un altro libro più recente, L'onda d'urto, ho fatto un altro esperimento narrativo, sovvertendo i generi tradizionali. Si tratta di racconti che, letti in un certo ordine, formano un romanzo a frammenti: sono le storie immaginate o sognate dai personaggi, rifugiatisi sugli Appennini in seguito a una catastrofe immane che avviene all'inizio, nel primo racconto. Da quelle

6 J. Monteiro Martins, madrelingua, Nardò (Lecce), Besa Editrice, 2005. 
montagne i personaggi scendono solo nella conclusione del libro, iniziando un viaggio verso il Sudamerica. Così, nella mia esperienza, a partire dalla rivoluzione del racconto breve, i generi letterari si sono fusi e mescolati, al punto che ogni nuova opera è un genere letterario a sé, non riducibile alle etichette tradizionali, insufficienti e utili ormai soltanto per divisioni in categorie didattiche senza significato reale.

Dopo questa lunga digressione - il tema del racconto breve mi appassiona come pochi altri - arriviamo alla terza lingua della memoria, la mia lingua madre per eccellenza, la lingua portoghese. Per me, fino all'adolescenza, era il centro linguistico del mondo, l'idioma fonte di tutto ciò che esisteva, il Verbo del principio. Mi viene in mente un'espressione che si usava, e forse si usa ancora in Brasile, con gli stranieri che faticavano a farsi capire in Portoghese. Dicevamo: «Fale em língua de gente, por favor», letteralmente «Parla lingua di gente, per favore». Ossia, tutte le altre lingue eccetto la nostra non erano per noi lingue umane, per le stesse ragioni per cui i greci antichi chiamavano i forestieri «barbari», cioè «balbuzienti». Io parlavo, e scrivevo, in «lingua di gente». Naturale, no? Era la stessa lingua che il poeta Fernando Pessoa riteneva la sua unica patria. I primi nove libri che ho pubblicato li ho scritti in Portoghese, ma in un certo senso, se vogliamo, anche i successivi, perché dietro il mio Italiano, la mia lingua della vita, del presente, ossia dell'unica porzione di tempo a cui mi è permesso di attingere sempre, echeggia fortemente la lingua madre della memoria. Con la sua musica e le sue metafore, l'accortezza dei suoi detti, le pause e le percussioni delle sue vocali, i sospiri che esala nelle consonanti mute, entra in un rapporto discreto con le parole straniere, spostando leggermente il loro senso come fa con la loro pronuncia. Cammina al loro fianco, gli sussurra qualcosa, la tira un po' per il braccio, madre e figlia en promenade. E si sa, la madre è sempre lì, non ci abbandona mai.

Succede tuttavia non di rado nella vita di una persona di perdere la madre in giovane età e di passare il resto della vita in compagnia dei fratelli e delle sorelle. Allo stesso modo si può perdere la lingua madre, per colpa di un esilio, di una migrazione. Col tempo essa diventerà un dolcissimo ricordo, ritornerà nei sogni ogni notte, mentre l'orfano trascorrerà il resto della sua esistenza in compagnia delle lingue sorelle. Questo concetto di «lingue sorelle» l'ho coniato perché non trovavo un modo migliore per esprimere una tendenza che mi sembra sempre più comune tra scrittori e non: avere un'altra lingua non originaria, ma coetanea, con cui si stabilisce un rapporto da adulto ad adulto, e che a partire da un punto qualsiasi della maturità del 
neoparlante l'accompagnerà fino alla sua fine - e poi penserà anche ai suoi figli -. Proprio come una brava sorella.

Ecco, la lingua italiana è la mia cara lingua sorella. A lei, tutti i giorni, racconto i miei segreti e le mie storie. E lei mi risponde, interpretando il mio pensiero con sempre maggior intimità. Fortunato lo scrittore che può contare su una tale splendida famiglia! 